\title{
nature
}

biotechnology

\section{Honing our reading skills}

\author{
Studies from the RNA Sequencing Quality Control (SEQC) initiative exemplify the kind of experimental groundwork \\ needed to expand RNA-seq into a broader array of basic and translational applications.
}

The he ability to comprehensively decipher the transcripts encoded by a genome is a long-held goal of biological research. Since bursting onstage in 2008, high-throughput sequencing of RNA (RNA-seq) has begun to supplant DNA microarray analysis as a gene expression assay technology because of its larger dynamic range and ability to discover new transcripts without any prior sequence knowledge. But the approach remains a work in progress. Not only has the dizzying pace of innovation in sequencing technology raised questions about the performance of different platforms in different experimental contexts, but the statistical approaches for deciphering sequencer output and the software for implementing them have also struggled to keep pace with the burgeoning diversity of RNA profiling experiments under way. This issue features a set of reports that begin to address these questions, but progress on many fronts will be needed for RNA-seq to fulfill its potential in both basic and clinical applications.

Until recently, microarrays were the technology of choice for general analysis of the transcriptome, although other techniques, such as cap analysis gene expression and expressed sequence tag sequencing, provided powerful alternatives offering advantages for specific applications. Three of the drawbacks of microarray technology were saturation of readout for highly expressed transcripts (due to analog-type fluorescent signal), a limited dynamic range in terms of detecting low-level transcripts and cross-hybridization of transcripts to different probes on the microarray, which can introduce false positives into measurements.

Although RNA-seq overcomes some of these problems, it has issues of its own. For example, certain short reads obtained from sequencing may align ambiguously with the reference genome. Another limitation is the tradeoff between a smaller number of long reads (which are good for resolving alternatively spliced transcript isoforms) and massive numbers of short reads, which allow analyses of genes but not necessarily transcripts. A third difficulty is that RNA molecules in a sample are effectively in competition with each other to be sequenced. As a result, highly abundant as well as longer transcripts will consume more sequencing resources, leaving less for other RNAs. All of which makes data collection and analysis of short-read output a complex problem.

Accounting for biological variation as well as technical variation has been a long-standing problem in gene expression analysis, predating RNA-seq's debut. With microarrays, it took several years from when the technology was first described until robust statistical approaches were established for modeling the data (Nat. Biotechnol. 29, 331-333, 2011). These models accounted for insights into the nature of the assay, such as dependencies between the variance and intensity of a measurement, and features of the technology, such as data from multiple probes per gene or from 'mismatch' probes not perfectly complementary to their target sequence. Moreover, it was recognized that analysis of microarray data could be confounded by batch effects owing to differences introduced by processing arrays at different times, in different locations or even by different technicians.

Several of the lessons learned from microarrays about the importance of accounting for technical variation and batch effects are being embraced by the research community in applying RNA-seq. Early studies identified GC biases in RNA-seq data, a relationship between read length and read depth (longer sequences generate more reads, resulting in greater statistical power to detect differential expression), and biases in the nucleotide composition of reads that arise from the use of random hexamers when converting RNA to cDNA.

The studies in this issue, carried out by the SEQC Consortium (pp. 903, 926 and 888) and a complementary project by the Association of Biomolecular Resource Facilities (ABRF; p. 915), investigate sources of bias and compare the performance of different RNA-seq approaches, primarily to address the question of whether sequencing the same samples at different facilities yields similar results. These efforts complement a recent study from the GEUVADIS consortium (Nat. Biotechnol. 31, 1015-1022, 2013), and the SEQC project is an extension of the MicroArray Quality Control (MAQC)-I (Nat. Biotechnol. 24, 1151-1161, 2006) and MAQC-II (Nat. Biotechnol. 28, 827-838, 2010) initiatives.

Reassuringly and perhaps expectedly, many of the same factors found in smaller-scale studies to influence RNA-seq were also observed by the SEQC Consortium and ABRF across multiple platforms. A notable aspect of the studies is the use of RNA spike-ins added to each sample to provide common points of reference for downstream data analysis. Although the spike-ins themselves are not new, the studies in this issue mark the first time that they have been used in a comprehensive evaluation of differential expression with RNA-seq. This facilitated the development of new tools for using spike-ins to evaluate sample quality (Munro, S. et al. Nat. Commun., in the press) and normalize data to remove unwanted variation (p. 896).

Going forward, RNA-seq is poised to transform biomedical research. It promises to expand our understanding of transcription in disease from simple measurement of gene expression level or known splice junctions (essentially all that can be done using microarrays) to measurement of transcript isoform levels. Furthermore, as future studies seek to understand variation in the genome and transcriptome in the context of clinical phenotypes, more samples from larger cohorts of individuals or single cells will be required to provide sufficient statistical power. The scale and cost of such efforts will likely place an even greater emphasis on research collaborations and consortia that carry out experiments at different centers.

This underscores the importance of the SEQC and ABRF studies published in these pages. The human transcriptome may not be an open book as yet. But these benchmark studies provide the ground work for more ambitious clinical studies. And they provide an important step in establishing the best practices for honing our transcriptome-reading skills. 